\title{
Lift on a Low Speed Circular Arc Wing due to Air Compression
}

\author{
Kern E. Kenyon \\ 4632 North Lane, Del Mar, USA
}

Correspondence to: Kern E. Kenyon, kernken@aol.com

Keywords: Slow Flight Lift, Air Compressibility

Received: February 7, $2021 \quad$ Accepted: March 20, $2021 \quad$ Published: March 23, 2021

Copyright $\odot 2021$ by author(s) and Scientific Research Publishing Inc.

This work is licensed under the Creative Commons Attribution International License (CC BY 4.0).

http://creativecommons.org/licenses/by/4.0/

\section{(c) (1) Open Access}

\section{ABSTRACT}

\begin{abstract}
A fluid flow model consisting of Bernoulli's law in its normal form, the equation of state of air, and the cross-stream force balance between a downward pressure gradient and the upward centrifugal force on fluid particles moving along curved streamlines over the top circular wing surface involving three equations in three unknowns (pressure, density and velocity) are solved to show that both density and pressure decrease upward as the inverse square of the distance from the circle's center, and the velocity is independent of that distance. These derived characteristics are used to explain the lift force on the wing in what is believed to be a novel way.
\end{abstract}

\section{INTRODUCTION}

Conceptually, there are two different ways Bernoulli's law can lead to an understanding of increasing the lift on a plane's wing. One way normally thought of is to arrange the relative speed of air flow to be greater on the top surface than on the bottom one, because where the speed is greatest, the pressure is least [1]. A second way, which I have never seen advanced, is to have greater air density above than below. Nobody goes around saying that where the air is heaviest, the pressure is least. But one could say that as will be shown below.

Compressibility is here explored as the suggested extension of the lift force calculation for a circular arc wing in a recent study [2], which contains a separate innovation but has a limitation as well, presumed to have been stemming mainly from the assumption of constant air density, and/or possibly the adopted spatial structure of the fluid velocity. Getting around the limitation motivated the present discussion.

It is not normal to consider compressibility in fluid flow problems that involve flow speeds much lower than that of sound. Here, the evolution unexpectedly came from the study of incompressible fluid flow structures that have no friction according to the Navier-Stokes equations in polar coordinates, like circular ones where the velocity is inversely proportional to the radius [3]. Also, an apparently unrelated result came from applying compressibility to the tornado [4]. These investigations and the present one are 
not burdened by an initial assumption of irrotationality, upon which so much earlier work on lift is founded.

Intuition may suggest that if a gas impinges on a rigid surface, a wing for example, compression may occur at and near that surface even at low relative speeds. In other words, there could be a boundary layer of compressed air attached to the wing's top surface. For some reason, the text books in fluid dynamics appear to try to steer the researcher of compressibility toward the high speeds. Shock waves are interesting, of course.

\section{METHOD}

Begin by collecting three equations in three variables: pressure $p$, velocity $u$ and density $\rho$. These same three equations have been gathered before but for a very different application: the tornado [4]. First Equation (1) is Bernoulli's equation along a streamline which does not include the available compressibility term [5]. Second is the equation of state for air [6]. Third equation is the cross-stream force balance on fluid particles between a downward pressure force and an upward centrifugal force for curved streamlines above the circular arc wing.

An explanation is in order as to why the form of Bernoulli's equation selected below does not have a compressibility term in it. Compressibility across streamlines is deemed more important for this problem than air density variations along individual streamlines.

$$
p=C-\frac{1}{2} \rho u^{2}
$$

where $C$ is a constant. Taking $C=0$ is not anticipated to significantly affect the dynamics of the problem.

$$
p=S \rho
$$

where $S=R T$ and $R$ is the gas constant for air and $T$ is the temperature assumed constant.

$$
\frac{\partial p}{\partial r}=\frac{\rho u^{2}}{r}
$$

where $r$ measures distance from the center of the circle of which the arc wing's surface is a part.

Between (1) and (3) the density and velocity can both be eliminated to get the pressure equation

$$
r \frac{\partial p}{\partial r}+2 p=0
$$

which has the solution

$$
p=\frac{\text { const }}{r^{2}}
$$

where the constant in the numerator needs to be evaluated. Another constant could be added to (5) if the constant $C$ in (1) were not set equal to zero.

From (2) and (5) the density is

$$
\rho=\frac{\text { const }}{S r^{2}}
$$

So both density and pressure decrease with increase of the radius as the inverse square. With this knowledge it can be shown from (1) that the velocity $u$ does not vary with $r$.

\section{DISCUSSION}

Given that the velocity $u$ does not vary with the radius $r$, suggested by the above method, but the density $\rho$ decreases as the inverse square of $r$, then the mass flux $\rho u$ over the top of the wing can be calculated, because its integral from the top of the wing to infinity will converge. Then by comparison with an 
analogous mass flux far away from the wing one of the constants can be evaluated. In this way the problem in the earlier study [1] can be avoided, where the air density was taken constant, the velocity decreased upward inversely with $r$, and the mass flux integral diverged. in (6)

For example, if the constant air density and relative flow speed away from the wing are: $\rho_{0}, U$, then

$$
\text { const }=\rho_{0} h r_{0} S
$$

where $h$ is the maximum thickness of the wing and $r_{0}$ is the radius of the circular arc's surface.

If the Schlieren imaging technique were to be applied to the air flowing over the top surface of a wing, perhaps density variations of the air could be detected which would strengthen the proposed lift concept.

\section{CONCLUSION}

According to the fluid flow model for air presented above, it is concluded that the lift force on a slowly translating circular arc wing can be related more to a boundary layer of compressed air attached to the wing's top surface than to the usual explanation of a greater mean flow speed above the wing compared to that below it.

\section{CONFLICTS OF INTEREST}

The author declares no conflicts of interest regarding the publication of this paper.

\section{REFERENCES}

1. Kenyon, K.E. (2017) Lift Force on a Circular Arc Wing. Natural Science, 9, 351-354. https://doi.org/10.4236/ns.2017.910033

2. Kenyon, K.E. (2020) Lift on a Circular Arc Wing II. European International Journal of Science and Technology, 9, 21-24.

3. Batchelor, G.K. (1967) An Introduction to Fluid Dynamics. Cambridge University Press, San Diego, 203.

4. Kenyon, K.E. (2020) Tornado's Compressibility. Natural Science, 12, 218-220. https://doi.org/10.4236/ns.2020.124019

5. Faber, T.E. (1995) Fluid Dynamics for Physicists. Cambridge University Press, Cambridge, UK, 86. https://doi.org/10.1017/CBO9780511806735

6. Brunt, D. (1952) Physical and Dynamical Meteorology. Cambridge University Press, New York, 30. 